\title{
Magnetocaloric effect and Grüneisen parameter of quantum magnets with a spin gap
}

Rakhimov, Abdulla ; Gazizulina, Alsu ; Narzikulov, Zabardast ; Schilling, Andreas ; Sherman, E Ya

\begin{abstract}
We obtained, within a path-integral formalism (mean-field variational Gaussian approximation), analytical expressions for thermodynamic quantities such as magnetization, heat capacity, and the magnetic Grüneisen parameter $\Gamma H$ of the system of triplons in spin gapped quantum magnets. Near the critical temperature $\Gamma \mathrm{H}$ is discontinuous and changes its sign upon the Bose-Einstein condensation (BEC) of triplons. We predict that in the limit of low temperature $\mathrm{T}$ and near the critical magnetic field $\mathrm{Hc}, \Gamma \mathrm{H}$ diverges as $\Gamma \mathrm{H} 1 / \mathrm{T} 2$, while it scales as $\Gamma \mathrm{H} 1 /(\mathrm{H}-\mathrm{Hc})$ as the magnetic field approaches Hc.
\end{abstract}

DOI: https://doi.org/10.1103/physrevb.98.144416

Posted at the Zurich Open Repository and Archive, University of Zurich

ZORA URL: https://doi.org/10.5167/uzh-159702

Journal Article

Published Version

Originally published at:

Rakhimov, Abdulla; Gazizulina, Alsu; Narzikulov, Zabardast; Schilling, Andreas; Sherman, E Ya (2018). Magnetocaloric effect and Grüneisen parameter of quantum magnets with a spin gap. Physical review. B, 98(14):144416.

DOI: https://doi.org/10.1103/physrevb.98.144416 


\title{
Magnetocaloric effect and Grüneisen parameter of quantum magnets with a spin gap
}

\author{
Abdulla Rakhimov, ${ }^{1}$ Alsu Gazizulina, ${ }^{2}$ Zabardast Narzikulov, ${ }^{3}$ Andreas Schilling, ${ }^{3}$ and E. Ya. Sherman ${ }^{4,5}$ \\ ${ }^{1}$ Institute of Nuclear Physics, Tashkent 100214, Uzbekistan \\ ${ }^{2}$ National University of Uzbekistan, Tashkent 100174, Uzbekistan \\ ${ }^{3}$ Physik-Institut, University of Zürich, Winterthurerstrasse 190, 8057 Zürich, Switzerland \\ ${ }^{4}$ Department of Physical Chemistry, The University of the Basque Country UPV/EHU, 48080 Bilbao, Spain \\ ${ }^{5}$ IKERBASQUE Basque Foundation for Science, 48013 Bilbao, Spain
}

(Received 31 May 2018; revised manuscript received 17 September 2018; published 10 October 2018)

\begin{abstract}
We obtained, within a path-integral formalism (mean-field variational Gaussian approximation), analytical expressions for thermodynamic quantities such as magnetization, heat capacity, and the magnetic Grüneisen parameter $\Gamma_{H}$ of the system of triplons in spin gapped quantum magnets. Near the critical temperature $\Gamma_{H}$ is discontinuous and changes its sign upon the Bose-Einstein condensation (BEC) of triplons. We predict that in the limit of low temperature $T$ and near the critical magnetic field $H_{c}, \Gamma_{H}$ diverges as $\Gamma_{H} \sim 1 / T^{2}$, while it scales as $\Gamma_{H} \sim 1 /\left(H-H_{c}\right)$ as the magnetic field approaches $H_{c}$.
\end{abstract}

DOI: 10.1103/PhysRevB.98.144416

\section{INTRODUCTION}

The properties of condensed matter at low temperatures have always been of high interest. Phenomena such as novel types of superconductivity/superfluidity, quantum phase transitions, or different types of topological order attract attention of a growing community of researchers. For condensed matter systems, P. Debye [1] and W. F. Giauque [2] independently suggested in 1926 to use the magnetocaloric effect (MCE) of paramagnetic materials to reach temperatures significantly below $1 \mathrm{~K}$. This effect, which describes the temperature changes of a magnetic material in response to an adiabatic variation of the magnetic field, forms the basis of magnetic refrigeration. The observation of a giant MCE around room temperature has stimulated additional work in this field (see recent review by Wolf et al. [3]).

The magnetocaloric effect and the related magnetic Grüneisen parameter,

$$
\Gamma_{H}=\frac{1}{T}\left(\frac{\partial T}{\partial H}\right)_{S}
$$

corresponding to the temperature gradient in the $T(H)$ landscape along an isoentropic (constant entropy $S$ ) line quantify the cooling or heating of a material when an applied magnetic field is changing while the entropy remains a constant. In such a process the exchanged heat is zero,

$$
\delta Q=T d S=T\left(\frac{\partial S}{\partial T}\right)_{H} d T+T\left(\frac{\partial S}{\partial H}\right)_{T} d H=0,
$$

and hence

$$
\Gamma_{H}=-\frac{1}{C_{H}}\left(\frac{\partial S}{\partial H}\right)_{T},
$$

where $C_{H}=T(\partial S / \partial T)_{H}$ is the heat capacity at constant magnetic field $H$. Experimentally, $\Gamma_{H}$ can be directly accessed by measuring the temperature at the constant entropy upon magnetic field variation and using Eq. (1). An equivalent expression for the Grüneisen parameter using the magnetization $M$,

$$
\Gamma_{H}=-\frac{1}{C_{H}}\left(\frac{\partial M}{\partial T}\right)_{H},
$$

can be derived by assuming a constant volume and pressure, from the grand thermodynamic potential $\Omega$ and suitable Maxwell relations using $d \Omega=-S d T-N d \mu-M d H$, with $\mu$ being the chemical potential and $N$ being the number of particles.

By using scaling analysis, Zhu et al. [4] concluded that the Grüneisen parameter is divergent at any quantum critical point (QCP), while Garst and Rosch considered the sign of $\Gamma_{H}$ across it [5]. They established the following features of the magnetic Grüneisen parameter $\Gamma_{H}$.

(1) Near the QCP $H_{c}$ at $T=0$, the distance to the QCP can be characterized by a dimensionless parameter $r(H)=$ $\left(H-H_{c}\right) / H_{c}$. Then

$$
\Gamma_{H}(T \rightarrow 0, r)=G_{r} \frac{1}{H-H_{c}},
$$

where $G_{r} \geqslant 0$ is a prefactor which can be related [4,5] to the critical correlation-length exponent $v$, the dynamical exponent $z$, and the dimensionality of the critical fluctuation $d$ as

$$
G_{r}=v(d-z) .
$$

For example, for the Ising chain in a transverse magnetic field one finds $G_{r}=1$, while a dilute Bose gas in the symmetrybroken phase should show $G_{r}=1 / 2$ [5].

(2) The temperature dependence of $\Gamma_{H}$ in the critical regime at low temperatures also shows a divergence,

$$
\Gamma_{H}\left(T, H \rightarrow H_{c}\right) \sim \frac{1}{T^{x}},
$$

with $x=1 / z v$. 
(3) It is predicted that $\Gamma_{H}$ exhibits a sign change at the QCP, so that the Grüneisen parameter has a different sign on each side of the phase transition.

These characteristic divergences and the sign change of the Grüneisen parameter are hallmarks to detect and identify putative quantum critical points.

These properties of $\Gamma_{H}$ have been experimentally confirmed by Gegenwart et al., who developed a low-frequency alternating-field technique to measure $\Gamma_{H}$ down to low temperatures [6], in order to classify a number of magnetic systems ranging from heavy-fermion compounds to frustrated magnets [7]. They indeed observed a universal scaling of Grüneisen parameter $\Gamma_{H}=G_{r}\left(H-H_{c}\right)^{-1}$ with $G_{r} \approx 0.3$ and $\Gamma_{H} \sim 1 / T^{x}$, with $x$ varying from 1 to 3 in a certain deviation from the Hertz-Millis theory prediction $x=1$ [8].

There is a class of materials referred to as zero field gap quantum magnets $[9,10]$. In a subclass of these materials containing dimers of two spin $1 / 2$ entities, the spin gap between excited triplet and singlet ground states closes beyond a critical magnetic field $H_{c}$ due to the Zeeman effect. As a result, bosonic quasiparticles ("triplons") arise, which may undergo a BEC below a critical temperature $T_{c}$. Although experimental data on thermodynamic properties are available for many of such systems (for a review, see Refs. [9] and [10]), measurements of the MCE and the associated Grüneisen parameter are rare $[9,11,12]$. Experimentally it is very difficult to explore the behavior of $\Gamma_{H}$ in the zero-temperature limit. This topic has not yet been systematically addressed for these materials, to the best of our knowledge, neither theoretically nor experimentally, with, perhaps, only a single exception [11].

From simple arguments, the property $\Gamma_{H} \sim 1 / T^{x}$ can be easily considered for noninteracting Bose systems using $C_{H}(T \rightarrow 0) \sim T^{3 / 2}$ and $M \sim M(0)-\left(T / T_{c}\right)^{3 / 2}$. From Eq. (4), all materials belonging to the noninteracting BEC universality class should therefore obey $\Gamma_{H} \sim 1 / T$, i.e., $x=$ 1 [11]. Nevertheless, as the triplon bosonic quasiparticles in the magnetic insulators to be considered here are known to constitute an interacting Bose gas [9,10,13], a consideration of the effects of interaction on the Grüneisen parameter is of utmost interest. The aim of the present work is to investigate the properties of $\Gamma_{H}$ for such magnets within a variational perturbation theory. We will show that the fractional exponent is $z v=1 / 2$ (i.e., $x=2), \Gamma_{H} \sim 1 /\left(H-H_{c}\right)$ and demonstrate that $\Gamma_{H}$ changes its sign at the transition.

Here and below we adopt the units $k_{\mathrm{B}} \equiv 1$ for the Boltzmann constant, $\hbar \equiv 1$ for the Planck constant, and $V=a^{3} \equiv$ 1 , where $a$ is the lattice constant, for the unit cell volume $V$. In these units the energies are measured in Kelvin, the mass $m$ is expressed in $1 / \mathrm{K}$, while the momentum, the entropy, and specific heat are dimensionless. Particularly, the Bohr magneton is $\mu_{B}=0.6717 \mathrm{~K} / \mathrm{T}$.

This paper is organized as follows. In Sec. II we derive main equations for thermodynamic quantities both in the normal and condensed phases. In Sec. III, applying long-wave approximation, we obtain low temperature expansion. In Sec. IV the critical behavior of $C_{H}$ and $\Gamma_{H}$ near $T_{c}$ will be studied. In Sec. V we present our numerical results and discussions and present analysis of experimental measurability on Sec. VI. Section VII includes the main conclusions. Some details of the calculations are given in the Appendices.

\section{THE FREE ENERGY AND ENTROPY OF THE TRIPLON GAS}

For $H>H_{c}$ the thermodynamics of a dimerized quantum magnet is determined by the system of triplon quasiparticles with integer spin if we neglect the phonon contribution for the moment. In a constant external magnetic field, the number of triplons is conserved in the thermodynamic limit, and they can experience Bose-Einstein condensation $[9,10,13,14]$. Although the critical temperature $T_{c}$ or the density of triplons of the BEC may be obtained within Hamiltonian formalism [15-18], it is more convenient to derive the thermodynamic potential in path integral formalism, by using variational perturbation theory [19].

In this formalism one starts with the action

$$
\begin{aligned}
\mathcal{A}\left[\psi^{\dagger}, \psi\right]= & \int_{0}^{\beta} d \tau \int d^{3} r\left\{\psi^{\dagger}\left[\frac{\partial}{\partial \tau}-\hat{K}-\mu\right] \psi\right. \\
& \left.+\frac{U}{2}\left(\psi^{\dagger} \psi\right)^{2}\right\},
\end{aligned}
$$

where $\beta \equiv 1 / T, \mu$ is the chemical potential, here given as $\mu=\mu_{B} g\left(H-H_{c}\right)$ with the Lánde $g$ factor $[9,10,13,14,20]$, and $\mu_{B}$ is the Bohr magneton. The operator of kinetic energy, $\hat{K}$ gives rise to the bare dispersion of triplons $\varepsilon_{\mathbf{k}}$ as defined, for example, in the bond operator representation $[21,22]$ or in the gap-dependent "relativistic" form of the spectrum [23]. The parameter $U$ represents a constant describing a strong shortrange triplon-triplon repulsion [24]. The complex fields, $\psi^{\dagger}$ and $\psi$ satisfy the standard bosonic periodicity conditions in that $\psi(\tau, \mathbf{r})$ and $\psi^{\dagger}(\tau, \mathbf{r})$ are periodic in $\tau$ with period $\beta$. The integration in the coordinate space may be taken within the crystal unit cell [25].

Then the thermodynamical potential $\Omega$ can be obtained from

$$
\Omega=-T \ln \mathcal{Z},
$$

where the grand-canonical partition function $\mathcal{Z}$ is given by the path integral [26]

$$
\mathcal{Z}=\int \mathcal{D} \psi^{\dagger} \mathcal{D} \psi e^{-A\left[\psi^{\dagger}, \psi\right]} .
$$

Due to the complications related to the $\left(\psi^{\dagger} \psi\right)^{2}$ term in (8), the path integral cannot be evaluated exactly. In the present work we shall use a variational perturbation theory [27] as outlined in Refs. [28] and [29] for finite systems. Referring the reader to Appendix A for the calculation details, we obtain for $\Omega$

$$
\begin{aligned}
\Omega= & \Omega_{\mathrm{cl}}+\Omega_{2}+\Omega_{4}, \\
\Omega_{\mathrm{cl}}= & -\mu_{0} \rho_{0}+\frac{U \rho_{0}^{2}}{2}+\frac{1}{2} \sum_{\mathbf{k}}\left(\mathcal{E}_{\mathbf{k}}-\varepsilon_{\mathbf{k}}\right) \\
& +T \sum_{\mathbf{k}} \ln \left[1-\exp \left(-\beta \mathcal{E}_{\mathbf{k}}\right)\right], \\
\Omega_{2}= & \frac{1}{2}\left[A_{1}\left(U \rho_{0}-X_{2}-\mu\right)+A_{2}\left(3 U \rho_{0}-X_{1}-\mu\right)\right], \\
\Omega_{4}= & \frac{U}{8}\left(3 A_{1}^{2}+2 A_{1} A_{2}+3 A_{2}^{2}\right),
\end{aligned}
$$


and for $(i, j)=(1,2)$ or $(2,1)$, respectively,

$$
\begin{aligned}
A_{i} & =\left.G_{j j}\left(\tau, \mathbf{r}, \tau^{\prime}, \mathbf{r}^{\prime}\right)\right|_{\mathbf{r} \rightarrow \mathbf{r}^{\prime}, \tau \rightarrow \tau^{\prime}} \\
& =T \sum_{n} \sum_{\mathbf{k}} \frac{\varepsilon_{\mathbf{k}}+X_{i}}{\omega_{n}^{2}+\mathcal{E}_{\mathbf{k}}^{2}}=\sum_{\mathbf{k}} \frac{\varepsilon_{\mathbf{k}}+X_{i}}{\mathcal{E}_{\mathbf{k}}} W_{\mathbf{k}},
\end{aligned}
$$

where

$$
\begin{aligned}
W_{\mathbf{k}} & =\frac{1}{2} \operatorname{coth}\left(\frac{\beta \mathcal{E}_{\mathbf{k}}}{2}\right)=\frac{1}{2}+f_{B}\left(\mathcal{E}_{\mathbf{k}}\right), \\
f_{B}(x) & =\frac{1}{e^{\beta x}-1},
\end{aligned}
$$

where $f_{B}(x)$ is the Bose distribution with

$$
\mathcal{E}_{\mathbf{k}}=\sqrt{\varepsilon_{\mathbf{k}}+X_{1}} \sqrt{\varepsilon_{\mathbf{k}}+X_{2}}
$$

being the dispersion relation of the quasiparticles. Here $X_{1}$ and $X_{2}$ are variational parameters defined from the principle of minimal sensitivity [19] as

$$
\frac{\partial \Omega\left(X_{1}, X_{2}, \rho_{0}\right)}{\partial X_{1}}=\frac{\partial \Omega\left(X_{1}, X_{2}, \rho_{0}\right)}{\partial X_{2}}=0 .
$$

The normal $\rho_{1}$ and the anomalous $\sigma$ densities become

$$
\begin{aligned}
& \rho_{1}=\int\left\langle\widetilde{\psi}^{\dagger} \widetilde{\psi}\right\rangle d^{3} r=\frac{A_{2}+A_{1}}{2}, \\
& \sigma=\int\langle\widetilde{\psi} \widetilde{\psi}\rangle d^{3} r=\frac{A_{2}-A_{1}}{2},
\end{aligned}
$$

respectively. Note that for the system considered here, it is the inclusion of the anomalous averages $\sigma$ [30], known as the Hartree-Fock-Bogoliubov approach, that allows one to obtain continuous magnetization across the BEC transition, which would be discontinuous otherwise, in the so-called HartreeFock-Popov approximation. The comparisons of these approaches and the results of numerical calculations are given in Ref. [15] (see Fig. 1 there for details) and in Ref. [16] (see Fig. 3 there), providing an approach to the system analysis equivalent to that presented here. Another model assuring the continuous behavior of the order parameter has been analyzed in Ref. [18].

From (11), (15), and (16) one obtains for $X_{1}$ and $X_{2}$

$$
\begin{gathered}
X_{1}=-\mu+U\left(2 \rho_{1}+3 \rho_{0}+\sigma\right), \\
X_{2}=-\mu+U\left(2 \rho_{1}+\rho_{0}-\sigma\right) .
\end{gathered}
$$

The stability condition $\partial \Omega / \partial \rho_{0}=0$ yields

$$
\mu_{0}-U \rho_{0}-2 U \rho_{1}-U \sigma=0,
$$

where $\rho_{0}$ is the condensed fraction summing up to the total density $\rho=\rho_{0}+\rho_{1}$, and $\mu_{0}$ is the corresponding Lagrange multiplier. In general, explicit expressions for all thermodynamic quantities can be inferred from $\Omega$ given in (11). In particular, differentiating $\Omega$ with respect to temperature yields the entropy

$$
\begin{aligned}
S= & -\left(\frac{\partial \Omega}{\partial T}\right)_{H}=-\sum_{\mathbf{k}} \ln \left[1-\exp \left(-\beta \mathcal{E}_{\mathbf{k}}\right)\right] \\
& +\beta \sum_{\mathbf{k}} \frac{\mathcal{E}_{\mathbf{k}}}{e^{\beta \mathcal{E}_{\mathbf{k}}}-1},
\end{aligned}
$$

while the heat capacity in constant magnetic field becomes

$$
C_{H}=T\left(\frac{\partial S}{\partial T}\right)_{H}=\beta^{2} \sum_{\mathbf{k}} \frac{\mathcal{E}_{\mathbf{k}}\left(\mathcal{E}_{\mathbf{k}}-T \mathcal{E}_{\mathbf{k}, T}^{\prime}\right) e^{\beta \mathcal{E}_{\mathbf{k}}}}{\left(e^{\beta \mathcal{E}_{\mathbf{k}}}-1\right)^{2}} .
$$

The resulting magnetic Grüneisen parameter is

$$
\Gamma_{H}=-\frac{g \mu_{B}}{C_{H}}\left(\frac{\partial S}{\partial \mu}\right)_{T}=\frac{\mu_{B} g \beta^{2}}{C_{H}} \sum_{\mathbf{k}} \frac{\mathcal{E}_{\mathbf{k}} \mathcal{E}_{\mathbf{k}, \mu}^{\prime} e^{\beta \mathcal{E}_{\mathbf{k}}}}{\left(e^{\beta \mathcal{E}_{\mathbf{k}}}-1\right)^{2}},
$$

where $\mathcal{E}_{\mathbf{k}, T}^{\prime}=\left(\partial \mathcal{E}_{\mathbf{k}} / \partial T\right)_{H}$ and $\mathcal{E}_{\mathbf{k}, \mu}^{\prime}=\left(\partial \mathcal{E}_{\mathbf{k}} / \partial \mu\right)_{T}$, given explicitly in Appendix B. Here and in what follows, summation over $\mathbf{k}$ implies the summation over the Brillouin zone: $\mathcal{B}=$ $\left\{-\pi \leqslant k_{\alpha} \leqslant \pi\right\}$, with $\alpha=x, y, z$. For further considerations, we discuss the normal $\left(T \geqslant T_{c}\right)$ and the condensed phase $\left(T<T_{c}\right)$ of the system separately.

\section{A. Normal phase, $T \geqslant T_{c}$}

When the temperature exceeds a critical temperature $T \geqslant$ $T_{c}$, the condensate fraction as well as the anomalous density vanish, i.e., $\rho_{0}=\sigma=0$, and $\rho_{1}=\rho$. Here the basic equations (17) and (18) have the same trivial solutions as

$$
X_{1}=X_{2}=2 U \rho-\mu .
$$

Inserting this into Eq. (14) gives

$$
\mathcal{E}_{\mathbf{k}}\left(T \geqslant T_{c}\right) \equiv \omega_{\mathbf{k}}=\varepsilon_{\mathbf{k}}-(\mu-2 U \rho) \equiv \varepsilon_{\mathbf{k}}-\mu_{\text {eff }},
$$

defining the effective chemical potential $\mu_{\text {eff }}$. Differentiating both sides of Eq. (24) with respect to $T$ and using Eq. (21) gives the following expression for the heat capacity:

$$
C_{H}\left(T \geqslant T_{c}\right)=\beta^{2} \sum_{\mathbf{k}} \frac{\omega_{\mathbf{k}} e^{\beta \omega_{\mathbf{k}}}\left(\omega_{\mathbf{k}}-2 U \rho_{T}^{\prime}\right)}{\left(e^{\beta \omega_{\mathbf{k}}}-1\right)^{2}} .
$$

The triplon density, which defines the longitudinal magnetization (i.e., the component parallel to $H$ ) via

$$
M=-\frac{\partial \Omega}{\partial H}=-\frac{\partial \Omega}{\partial \mu} \frac{\partial \mu}{\partial H}=\mu_{B} g \rho,
$$

is given by the solution of the nonlinear equation

$$
\begin{aligned}
\rho(T)=\rho_{1} & =\sum_{\mathbf{k}} \frac{1}{e^{\beta \omega_{\mathbf{k}}}-1} \\
& =\sum_{\mathbf{k}} \frac{1}{e^{\beta\left(\varepsilon_{\mathbf{k}}-\mu+2 U \rho\right)}-1},
\end{aligned}
$$

where we used Eqs. (12), (16), and (23). Note that in this phase, the staggered magnetization $M_{\perp}$, which is a hallmark for the BEC state in dimerized spin systems, vanishes. For the Grüneisen parameter we have from Eqs. (4) and (26)

$$
\Gamma_{H}\left(T>T_{c}\right)=-\frac{g \mu_{B}}{C_{H}} \rho_{T}^{\prime},
$$

where $\rho_{T}^{\prime}=\partial \rho / \partial T$ may be obtained from Eq. (27) (see Appendix B).

The critical density $\rho_{c}$, i.e., the density of quasiparticles at the critical temperature $T_{c}$, is reached as soon the effective chemical potential $\mu_{\text {eff }}$ vanishes, and hence

$$
\rho_{c}=\rho\left(T_{c}\right)=\frac{\mu}{2 U} .
$$


With this condition we may obtain the critical temperature as the solution of the equation

$$
\frac{\mu}{2 U}=\sum_{\mathbf{k}} \frac{1}{e^{\varepsilon_{\mathbf{k}} / T_{c}}-1},
$$

which will later be used to optimize the input parameters of the model by comparing experimental data with the calculated $T_{c}(H)$ dependence. Note that for atomic gases with $\varepsilon_{\mathbf{k}}=$ $k^{2} / 2 m$, the momentum integration in (30) yields the well known result [31]

$$
T_{c}^{0}=\frac{2 \pi}{m}\left(\frac{\mu}{2 U \zeta(3 / 2)}\right)^{2 / 3},
$$

where $\zeta(x)$ is the Riemann function.

\section{B. Condensed phase, $T<T_{c}$}

In the condensed phase where the $U(1)$ symmetry is spontaneously broken, one has to implement the Hugenholtz-Pines [32] theorem relating the normal and the anomalous self energies $\Sigma_{\mathrm{n}}$ and $\Sigma_{\mathrm{an}}$ to each other, i.e.,

$$
\Sigma_{\mathrm{n}}-\Sigma_{\mathrm{an}}=\mu .
$$

In our notation this leads to the equation [18]

$$
X_{2}=\Sigma_{\mathrm{n}}-\Sigma_{\mathrm{an}}-\mu=0,
$$

or

$$
\mu-U\left(2 \rho_{1}+\rho_{0}-\sigma\right)=0,
$$

where we have used Eq. (18). Due to Hugenholtz- Pines theorem, the excitation energy of the Bogoliubov mode becomes gapless [33],

$$
\mathcal{E}_{\mathbf{k}}\left(T<T_{c}\right) \equiv E_{\mathbf{k}}=\sqrt{\varepsilon_{\mathbf{k}}+X_{1}} \sqrt{\varepsilon_{\mathbf{k}}} .
$$

For the BEC of triplons with the bare effective mass $m$, one obtains $\mathcal{E}_{\mathbf{k}}=c k+O\left(k^{2}\right)$, where $c=\sqrt{\Delta / m}$ is the velocity of the first sound and $\Delta=X_{1} / 2$. At a larger momentum, where $\varepsilon_{\mathbf{k}} \gg c k$, one obtains $\mathcal{E}_{\mathbf{k}} \approx \varepsilon_{\mathbf{k}}$.

Eliminating $\rho_{0}=\rho-\rho_{1}$ from Eqs. (17) and (34) one obtains the basic equation

$$
\Delta=\mu+2 U\left(\sigma-\rho_{1}\right),
$$

where

$$
\begin{gathered}
\sigma=-\Delta \sum_{\mathbf{k}} \frac{W_{\mathbf{k}}}{E_{\mathbf{k}}}, \\
\rho_{1}=\sum_{\mathbf{k}}\left[\frac{W_{\mathbf{k}}\left(\varepsilon_{\mathbf{k}}+\Delta\right)}{E_{\mathbf{k}}}-\frac{1}{2}\right],
\end{gathered}
$$

and

$$
E_{\mathbf{k}}=\sqrt{\varepsilon_{\mathbf{k}}} \sqrt{\varepsilon_{\mathbf{k}}+2 \Delta},
$$

(see Ref. [34] for the origin of the term $1 / 2$ in (38)). Equation (34) with $\rho_{0}=\sigma=0$ gives the same expression for the critical density $\rho_{c}=\rho\left(T_{c}\right)=\mu / 2 U$ as in Eq. (29), which proves the self consistency of present approach. Taking $\partial E_{\mathbf{k}} / \partial T \equiv$ $E_{\mathbf{k}, T}^{\prime}$ from both sides of Eq. (39) with $E_{\mathbf{k}, T}^{\prime}=\varepsilon_{\mathbf{k}} \Delta_{T}^{\prime} / E_{\mathbf{k}}$ and inserting it into Eq. (21) gives

$$
C_{H}\left(T<T_{c}\right)=\beta^{2} \sum_{\mathbf{k}} \frac{e^{\beta E_{\mathbf{k}}}\left(E_{\mathbf{k}}^{2}-T \varepsilon_{\mathbf{k}} \Delta_{T}^{\prime}\right)}{\left(e^{\beta E_{\mathbf{k}}}-1\right)^{2}},
$$

where $\Delta_{T}^{\prime}$ is given in Appendix B.

For practical calculations Eq. (36) can be rewritten as

$$
Z=1+\widetilde{\sigma}(Z)-\tilde{\rho}_{1}(Z)
$$

where $Z=\Delta / \mu, \widetilde{\sigma}=\sigma / \rho_{c}$, and $\widetilde{\rho}_{1}=\rho_{1} / \rho_{c}$. After solving equation (41), the longitudinal and the staggered magnetizations $M$ and $M_{\perp}$ in the condensed phase, respectively, become

$$
\begin{aligned}
M\left(T \leqslant T_{c}\right) & =g \mu_{B} \rho=g \mu_{B} \rho_{c}(Z+1), \\
M_{\perp}^{2}\left(T \leqslant T_{c}\right) & =\frac{1}{2} g^{2} \mu_{B}^{2} \rho_{0}=\frac{1}{2} g^{2} \mu_{B}^{2} \rho_{c}(2 Z-\tilde{\sigma}),
\end{aligned}
$$

where we used

$$
\rho=\frac{\Delta+\mu}{2 U}, \quad \rho_{0}=\frac{\Delta}{U}-\sigma .
$$

The Grüneisen parameter is with Eqs. (42) and (43)

$$
\Gamma_{H}\left(T \leqslant T_{c}\right)=-\frac{g \mu_{B} \rho_{T}^{\prime}}{C_{H}}=-\frac{g \mu_{B} \Delta_{T}^{\prime}}{2 U C_{H}},
$$

where $C_{H}$ is given in Eq. (40).

\section{LOW TEMPERATURE EXPANSION}

In the present section we will derive analytical expressions in the $T \rightarrow 0$ limit. We shall perform the low-temperature expansion as a function of the dimensionless parameter $\widetilde{T} \equiv$ $T m$. In fact, for the majority of spin gap quantum magnets, the effective mass $m$ is small, e.g., $m \approx 0.02 \mathrm{~K}^{-1}$ for $\mathrm{TlCuCl}_{3}$ $[22,35]$, so that power series in the small parameter $\widetilde{T}$ quickly converges.

In general, three-dimensional momentum integrals, e.g., in Eqs. (37) and (38) cannot be evaluated analytically. To overcome this difficulty we use Debye-like approximation [36] and replace the Brillouin zone by the Debye sphere with the radius $k_{\mathrm{D}}$ such that for a regular function $\kappa(k)$ one obtains

$$
\begin{aligned}
\sum_{\mathbf{k} \in \mathcal{B}} \kappa(k) & =\frac{1}{(2 \pi)^{3}} \int_{\mathcal{B}} \kappa(k) d k_{x} d k_{y} d k_{z} \\
& =\int_{\mathcal{B}} \kappa(q) d q_{x} d q_{y} d q_{z} \approx \frac{\pi}{2} \int_{0}^{Q} q^{2} \kappa(q) d q .
\end{aligned}
$$

The normalization condition with $\kappa(k) \equiv 1$ yields dimensionless $Q=(6 / \pi)^{1 / 3}$ with $k_{\mathrm{D}}=Q \pi$ and $\mathbf{q} \equiv \mathbf{k} / \pi$. We assume simple symmetric three-dimensional bare dispersion

$$
\varepsilon_{\mathbf{k}}=J_{0}\left(3-\cos k_{x}-\cos k_{y}-\cos k_{z}\right),
$$

which we will use for numerical calculations as a model relation in gapped quantum magnets [10]. This bare dispersion enters Eqs. (14) and (35) for the spectrum of the Bogoliubov mode. At small $q$, we approximate it as $\varepsilon_{q} \approx q^{2} \pi^{2} / 2 m=$ $J_{0} k^{2} / 2$. This approximation can be used at low temperatures, while the effects of the spectrum nonparabolicity at higher temperatures [23] can be captured by the exact form of $\varepsilon_{\mathbf{k}}$ in Eq. (46). 
For the phonon dispersion we use long-wave approximation [36]:

$$
E_{q}=\sqrt{\varepsilon_{q}} \sqrt{\varepsilon_{q}+2 \Delta} \approx \pi c_{0} q
$$

with the sound velocity at zero temperature $c_{0}=\sqrt{\Delta_{0} / m}$, where $\Delta_{0}$ is the value of $\Delta$ at $T=0$. With these approximations for the low-temperature limit, most of the integrals can be evaluated explicitly in terms of logarithmic and polylogarithmic functions $\operatorname{Li}_{s}(z)$ of the argument $z=\exp \left(-\pi Q c_{0} \beta\right)$, i.e., as a function $F(T, z)$ [37]. Since $z$ decreases quickly with increasing $\beta$ we may expand $F(T, z)$ in powers of $z$ to extract the leading terms.

We refer the reader to Appendix B for the further details of the calculation. The result for the entropy becomes

$$
S=\frac{2 \pi^{2} \widetilde{T}^{3}}{45 \gamma^{3}}+O\left(\widetilde{T}^{5}\right)
$$

where $\gamma=c_{0} m=1 / \sqrt{2} \xi$ and $\xi$ is the healing length [38]. The derivative of (48) with respect to $T$ gives the heat capacity

$$
C_{H}=T \frac{\partial S}{\partial T} \approx \frac{2 \pi^{2} T^{3}}{15 c_{0}^{3}},
$$

which is common for interacting BEC systems since its measurement in superfluid helium [39]. Note that for an ideal Bose gas, i.e., for a system of noninteracting particles, the dispersion is not linear but quadratic, and $C_{H} \sim T^{3 / 2}$ [40].

To find an expression for the Grüneisen parameter, we use Eq. (42) with the relations

$$
\Gamma_{H}=-\frac{1}{C_{H}}\left(\frac{\partial M}{\partial T}\right)_{H}=-\frac{g \mu_{B}}{C_{H}}\left(\frac{\partial \rho}{\partial T}\right)_{H}=-\frac{g \mu_{B}}{2 U C_{H}} \Delta_{T}^{\prime} .
$$

The expansion for $\Delta_{T}^{\prime}$ becomes

$$
\Delta_{T}^{\prime}=-\alpha_{1} \widetilde{T}-\alpha_{3} \widetilde{T}^{3}+O\left(\widetilde{T}^{5}\right)
$$

where

$$
\begin{gathered}
\alpha_{1}=\frac{2}{3} \frac{U \pi \gamma}{U Q \gamma+\pi c_{0}}, \\
\alpha_{3}=\frac{U \pi^{2}}{45 \gamma^{3}} \frac{6 U Q \pi \gamma+6 \pi^{2} c_{0}+5 U \gamma^{2}}{\left(U Q \gamma+\pi c_{0}\right)^{2}} .
\end{gathered}
$$

Inserting $C_{H}$ from (49) we find

$$
\Gamma_{H}=\frac{15 g \mu_{B} \alpha_{1} \gamma^{2}}{4 \pi^{2} U} \frac{1}{\widetilde{T}^{2}}+\frac{15 g \mu_{B}\left(2 \gamma \alpha_{3}-\alpha_{1}^{2}\right)}{8 U \pi^{2} \gamma}+O\left(\widetilde{T}^{2}\right) .
$$

This is one of the central results of our paper. We will further simplify and discuss it later in Sec. V.

After using Eqs. (42), (B11), and (B12), the low temperature expansions for the magnetizations become

$$
M=g \mu_{B} \rho(T)=M(0)-\frac{g \mu_{B} \alpha_{1}}{4 U \gamma m} \widetilde{T}^{2}+O\left(\widetilde{T}^{4}\right)
$$

and

$$
M_{\perp}^{2}=M_{\perp}^{2}(0)-\frac{g^{2} \mu_{B}^{2} c_{0}\left(3 \alpha_{1}+U m\right)}{24 U \gamma^{2}} \widetilde{T}^{2}+O\left(\widetilde{T}^{4}\right) .
$$

Both quantities vary as $-T^{2}$ in the low-temperature limit while for a noninteracting Bose Einstein condensate, we would have a $-T^{3 / 2}$ dependence.

\section{PROPERTIES NEAR $T_{c}$}

The behavior of thermodynamic quantities in the temperature region $T \rightarrow T_{c} \pm 0$ is crucial for the nature of a phase transition. According to the Ehrenfest classification, a discontinuity in a second derivative of $\Omega$ at $T_{c}$ with a continuous first derivative indicates that the transition is of the second order [41]. In the present section we will study $C_{H}^{( \pm)} \equiv$ $C_{H}\left(T_{c} \pm 0\right), \Gamma_{H} \equiv \Gamma_{H}\left(T_{c} \pm 0\right)$, and $S^{( \pm)} \equiv S^{( \pm)}\left(T_{c} \pm 0\right)$

\section{A. $T \rightarrow T_{c}+0$ region}

Here $\mathcal{E}_{\mathbf{k}}=\omega_{\mathbf{k}}=\varepsilon_{\mathbf{k}}$ and $\mu=2 U \rho$. From Eqs. (25) and (B1)-(B3) we have

$$
C_{H}^{(+)}=-S_{3}+2 U S_{1} \rho_{T}^{\prime} .
$$

Here and below the functions $S_{1}(T), \ldots, S_{5}(T)$ given in Appendix B are evaluated at $T=T_{c}=1 / \beta_{c}$ as:

$$
\begin{aligned}
& S_{1}=-\beta_{c} \sum_{\mathbf{k}} \frac{\varepsilon_{\mathbf{k}} e^{\beta_{c} \varepsilon_{\mathbf{k}}}}{\left(e^{\beta_{c} \varepsilon_{\mathbf{k}}}-1\right)^{2}}, \\
& S_{2}=-U \beta_{c} \sum_{\mathbf{k}} \frac{e^{\beta_{c} \varepsilon_{\mathbf{k}}}}{\left(e^{\beta_{c} \varepsilon_{\mathbf{k}}}-1\right)^{2}}, \\
& S_{3}=-\beta_{c}^{2} \sum_{\mathbf{k}} \frac{\varepsilon_{\mathbf{k}}^{2} e^{\beta_{c} \varepsilon_{\mathbf{k}}}}{\left(e^{\beta_{c} \varepsilon_{\mathbf{k}}}-1\right)^{2}},
\end{aligned}
$$

and

$$
\rho_{T}^{\prime}=\frac{\beta_{c} S_{1}}{2 S_{2}-1} .
$$

It can be shown that

$$
\lim _{T \rightarrow T_{c}+0} \rho_{T}^{\prime}=0,
$$

since in this limit $S_{2}$ in the denominator of Eq. (59) has an infrared divergence at small $k$ while the nominator remains finite. Thus, Eq. (57) becomes

$$
C_{H}^{(+)}=-S_{3}=\beta_{c}^{2} \sum_{\mathbf{k}} \frac{\varepsilon_{\mathbf{k}}^{2} e^{\beta_{c} \varepsilon_{\mathbf{k}}}}{\left(e^{\beta_{c} \varepsilon_{\mathbf{k}}}-1\right)^{2}} .
$$

From Eq. (60) we may immediately conclude that the Grüneisen parameter at $T=T_{c}$ vanishes,

$$
\begin{aligned}
\Gamma_{H}^{(+)} & =-\frac{1}{C_{H}^{(+)}} \lim _{T \rightarrow T_{c}+0}\left(\frac{\partial M}{\partial T}\right)_{H} \\
& =-\frac{g \mu_{B}}{C_{H}^{(+)}} \lim _{T \rightarrow T_{c}+0}\left(\frac{\partial \rho}{\partial T}\right)_{H}=0,
\end{aligned}
$$

in agreement with the prediction of Garst et al. [5]. The entropy in Eq. (20) with $\mathcal{E}_{\mathbf{k}}=\varepsilon_{\mathbf{k}}$ is given by

$$
S^{(+)}=-\sum_{\mathbf{k}} \ln \left[1-\exp \left(-\beta_{c} \varepsilon_{\mathbf{k}}\right)\right]+\beta_{c} \sum_{\mathbf{k}} \frac{\varepsilon_{\mathbf{k}}}{e^{\beta_{c} \varepsilon_{\mathbf{k}}}-1} .
$$




\section{B. $T \rightarrow T_{c}-0$ region}

Here $\rho_{0}=0, \sigma=0$, and $\Delta=0$ and hence $\mathcal{E}_{\mathbf{k}}=E_{\mathbf{k}}=\varepsilon_{\mathbf{k}}$ again, i.e., the dispersion is the same on both sides of the critical temperature. For this reason the entropy is continuous at $T=T_{c}, S^{(-)}=S^{(+)}$. The heat capacity and the Grüneisen parameter are

$$
\begin{gathered}
C_{H}^{(-)}=\beta_{c}^{2} \sum_{\mathbf{k}} \frac{\varepsilon_{\mathbf{k}}\left(\varepsilon_{\mathbf{k}}-T_{c} \Delta_{T}^{\prime}\right) e^{\beta_{c} \varepsilon_{\mathbf{k}}}}{\left(e^{\beta_{c} \varepsilon_{\mathbf{k}}}-1\right)^{2}}, \\
\Gamma_{H}^{(-)}=-\frac{g \mu_{B} \Delta_{T}^{\prime}}{2 U C_{H}^{(-)}},
\end{gathered}
$$

where we used the relation $\rho_{T}^{\prime}=\Delta_{T}^{\prime} / 2 U$. The $\Delta_{T}^{\prime}$ defined in (B7) may be rewritten as

$$
\begin{aligned}
\Delta_{T}^{\prime} & =\frac{\beta_{c} U S_{4}}{2\left(2 S_{5}+1\right)}, \\
S_{4} & =-4 \beta_{c} \sum_{\mathbf{k}} \frac{\varepsilon_{\mathbf{k}} e^{\beta_{c} \varepsilon_{\mathbf{k}}}}{\left(e^{\beta_{c} \varepsilon_{\mathbf{k}}}-1\right)^{2}}, \\
S_{5} & =-U \beta_{c} \sum_{\mathbf{k}} \frac{T_{c}+\left(\varepsilon_{\mathbf{k}}-T_{c}\right) e^{\beta_{c} \varepsilon_{\mathbf{k}}}}{\varepsilon_{\mathbf{k}}\left(e^{\beta_{c} \varepsilon_{\mathbf{k}}}-1\right)^{2}} .
\end{aligned}
$$

Now we are in the position of evaluating discontinuities in $C_{H}$ and $\Gamma_{H}$. From Eqs. (61), (62), and (64) we can express them as

$$
\left[\Delta C_{H}\right]=C_{H}^{(-)}-C_{H}^{(+)}=-\beta_{c} \sum_{\mathbf{k}} \frac{\varepsilon_{\mathbf{k}} \Delta_{T}^{\prime} e^{\beta_{c} \varepsilon_{\mathbf{k}}}}{\left(e^{\beta_{c} \varepsilon_{\mathbf{k}}}-1\right)^{2}}>0,
$$

and

$$
\left[\Delta \Gamma_{H}\right]=\Gamma_{H}^{(-)}-\Gamma_{H}^{(+)}=-\frac{g \mu_{B} \Delta_{T}^{\prime}}{2 U C_{H}^{(-)}}>0,
$$

where $\Delta_{T}^{\prime}$ and $C_{H}^{(-)}$are given in (66) and (64), respectively, and $[\Delta \ldots]$ stands for the discontinuity of the corresponding quantity. From Eqs. (67) and (68) it is clear that not only $C_{H}$ but also the Grüneisen parameter has a finite jump near the critical temperature, and therefore the transition is of the second order according to the Ehrenfest classification.

It is easy to show that our results satisfy self-consistently the Ehrenfest relation

$$
\left[\Delta C_{H}\right]=-\left.\left.T_{c}\left(\frac{\partial H_{c}}{\partial T}\right)\right|_{T=T_{c}}\left[\Delta\left(\frac{\partial M}{\partial T}\right)\right]\right|_{T=T_{c}} .
$$

Using equations (30), (42), (60), and (67) leads to a modified Ehrenfest relation for the discontinuity in the Grüneisen parameter in triplon systems:

$$
\left[\Delta \Gamma_{H}\right]=\frac{\left[\Delta C_{H}\right]}{T_{c} C_{H}^{(-)}\left(\partial H_{c} / \partial T\right)},
$$

which can be derived from Eqs. (30), (64), (67), and (68).

\section{NUMERICAL RESULTS AND DISCUSSION}

\section{A. Sign change of $\Gamma_{H}$ at $T_{c}$}

In the previous section, we have shown that $\Gamma_{H}^{(+)}=0$ at the critical temperature $T_{c}$. It is also easy to show that $\Gamma_{H}(T)$ must change its sign there. Using Eqs. (62) and (B3) we have with $\partial \rho / \partial T>0$ and $\Gamma_{H}(T)<0$ for $T>T_{c}$. Approaching the critical temperature from below where $\partial \rho / \partial T<0$ [see Eq. (65)], $\Gamma_{H}(T)>0$ for $T<T_{c}$. This change of the sign in $\Gamma_{H}(T)$ has been conjectured by Garst et al. [5] to be universal for systems with a magnetically controlled critical point, which is also present at $H_{c}$ in the triplon systems considered here.

\section{B. Divergence of $\Gamma_{H}$ near the transition}

Rewriting the low temperature expansion of $\Gamma_{H}$ given in Eq. (54) in the limit $r=\left(H-H_{c}\right) / H_{c} \rightarrow 0$ in a compact form (see Appendix C), we obtain

$$
\Gamma_{H} \approx \frac{G_{t}\left(H-H_{c}\right)}{T^{2}}+\frac{G_{r}}{H-H_{c}},
$$

with

$$
G_{t}=\frac{5 g^{2} \mu_{B}^{2}}{\pi^{2}\left(1+4 a_{s} Q\right)^{2}} G_{r}
$$

and

$$
G_{r}=\frac{2}{\pi Q}+\frac{2}{U m Q^{2}} \simeq 0.51+\frac{0.1}{a_{s}},
$$

where the next higher-order terms are $O\left(\widetilde{T}^{2}\right)$ and $O(r)$, respectively. Here we used the relation

$$
U=\frac{4 \pi a_{s}}{m},
$$

where $a_{s}$ is the $s$-wave scattering length. The first term in Eq. (71) dominates $\Gamma_{H}(T, H)$ in a fixed magnetic field $H>H_{c}$ for temperatures $T \ll \eta\left(H-H_{c}\right)$, with $\eta \approx 0.48 g /\left(1+5 a_{s}\right.$ ) (in units of $\mathrm{K} / \mathrm{T}$ ), while the second term dominates in the opposite limit when $H$ approaches $H_{c}$ from above at a fixed low temperature $T$.

The divergence of $\Gamma_{H} \sim 1 / T^{2}$ at low enough temperatures for quantum spin gap magnets is one of our central results. Note that the classification of a number of magnetic systems ranging from heavy-fermion compounds to frustrated magnets done by Gegenwart et al. [7] reveals that the majority of them shows indeed a similar behavior, with some exceptions such as in Ref. [11], however. The fact that the critical parameters are sensitive to the dimensionality and other properties of a system make them belonging to different universality classes. The phase boundary between the condensed and the uncondensed states in spin gapped quantum magnets can be expressed by a power law of the form $T_{c} \propto$ $\left(H-H_{c}\right)^{1 / \phi\left(T_{c}\right)}$, with the scaling analysis of the quantum phase transitions predicting $1 / \phi\left(T_{c} \rightarrow 0\right)=z v$ [42]. The $T_{c}$ dependence of $\phi$ can appear due to the nonparabolic bare dispersion of triplons $[22,23]$ and is taken into account in our calculation using in Eq. (46). This nonparabolicity, however, does not modify qualitative features of the thermodynamic quantities.

The behavior $\Gamma_{H} \simeq G_{r}\left(H-H_{c}\right)^{-1}$, being well established in Refs. [5] and [7], is obviously also realized in the systems of the present work [see Eq. (71)]. We note, however, that this relation cannot be directly applied to the continuous systems such as atomic gases where $Q \rightarrow \infty$. In this case, renormalization procedures may lead to different dependences. 
We now briefly discuss whether the dimensionless parameter $G_{r}$ given in Eq. (73) is universal for triplon systems. For this purpose we note that two quantum magnets have the same $G_{r}$ if they have the same $a_{s}$ and $G_{r}$ approaches the universal 1/2 value, predicted by Garst et al. [5] in the unitary limit: $1 / a_{s} \rightarrow 0$. However, it should be mentioned that, in real systems, due to a possible anisotropy [18] the energy spectrum may deviate from that given by Eq. (39), and hence, the simple isotropic long wave approximation might be violated.

\section{Numerical results for realistic systems}

In the previous sections, we have given general expressions for $\Gamma_{H}, S, C_{H}, M$, and $M_{\perp}^{2}$, and elaborated the limiting cases $T \rightarrow 0$ and $T \rightarrow T_{c}$. We can use these results to numerically evaluate, using the bare dispersion presented in Eq. (46), these quantities over the full range of temperatures. In the following we will restrict ourselves to $\Gamma_{H}$ and $S$. To do this, we assume a set of realistic material parameters $g, H_{c}, U$, and $J_{0}$, which we take from experimental data for $\mathrm{Ba}_{3} \mathrm{Cr}_{2} \mathrm{O}_{8}, \mathrm{Sr}_{3} \mathrm{Cr}_{2} \mathrm{O}_{8}$, and $\mathrm{TlCuCl}_{3}[13,22,43-46]$ (see Table I).

To begin with, we show in Fig. 1 the $T_{c}$ dependence calculated from Eq. (30) for $\mathrm{Ba}_{3} \mathrm{Cr}_{2} \mathrm{O}_{8}$ and $\mathrm{Sr}_{3} \mathrm{Cr}_{2} \mathrm{O}_{8}$, together with experimental data taken from Refs. [43-45]. A clear deviation of the experimental points from $\phi=3 / 2$ dependence in
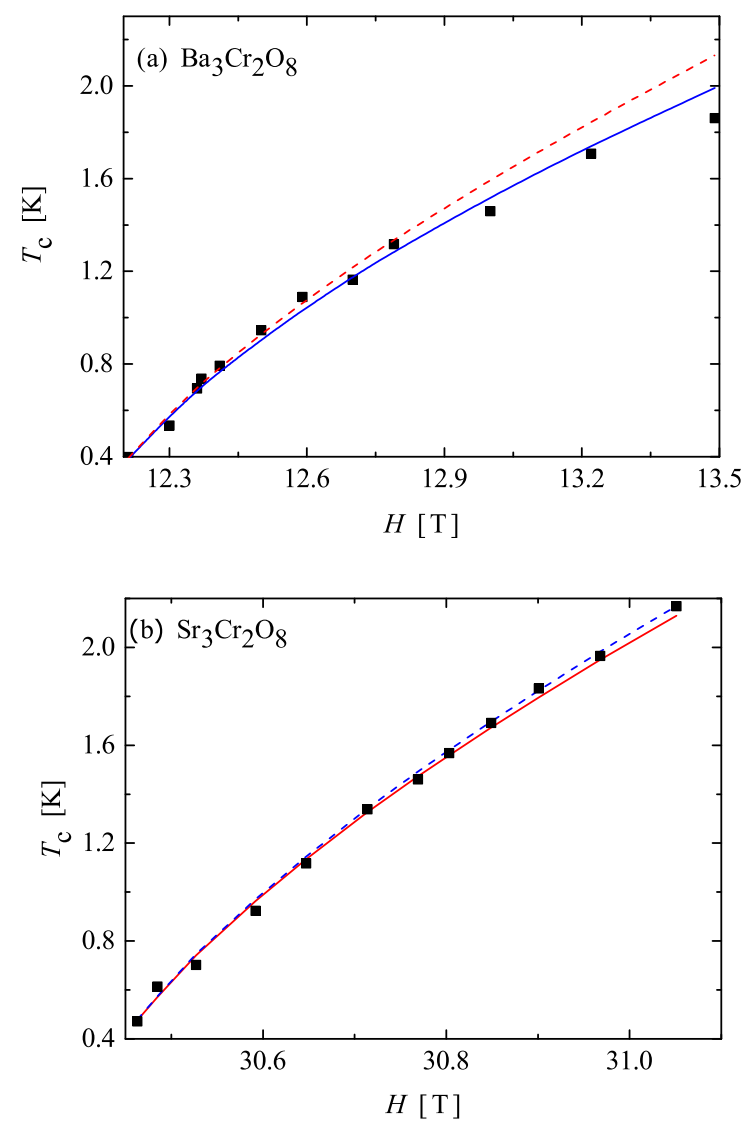

FIG. 1. The dependence of $T_{c}$ on the external magnetic field $H$ for model compounds (a) $\mathrm{Ba}_{3} \mathrm{Cr}_{2} \mathrm{O}_{8}$ and (b) $\mathrm{Sr}_{3} \mathrm{Cr}_{2} \mathrm{O}_{8}$ [solid lines from Eq. (30)]. The dashed lines correspond to the $1 / \phi=2 / 3$ behavior given in (31). The experimental data are taken from Ref. [44] (a) and Refs. [43] and [45] (b).
TABLE I. Material parameters used for our numerical calculations. From the input parameters $g, H_{c}$, and $U$ we derived $J_{0}$ from fitting the experimental phase boundary $T_{c}(H)$ to Eq. (30). $\Delta_{\mathrm{st}}$ corresponds to the energy scale of $H_{c}$ in Kelvin, while $G_{r}$ and $a_{s}$ come from Eqs. (73) and (74).

\begin{tabular}{lccccccl}
\hline \hline & $g$ & $H_{c}[\mathrm{~T}]$ & $J_{0}[\mathrm{~K}]$ & $U[\mathrm{~K}]$ & $\Delta_{\text {st }}[\mathrm{K}]$ & $G_{r}$ & \multicolumn{1}{c}{$a_{s}$} \\
\hline $\mathrm{Ba}_{3} \mathrm{Cr}_{2} \mathrm{O}_{8}$ & 1.95 & 12.10 & 5.045 & 20 & 15.85 & 0.84 & 0.315 \\
$\mathrm{Sr}_{3} \mathrm{Cr}_{2} \mathrm{O}_{8}$ & 1.95 & 30.40 & 15.86 & 51.2 & 39.8 & 0.9 & 0.257 \\
$\mathrm{TlCuCl}_{3}$ & 2.06 & 5.1 & 50. & 315 & 7.1 & 0.72 & 0.5 \\
\hline \hline
\end{tabular}

Fig. 1(a) is attributed to the nonparabolic bare energy at small $J_{0}$. For our calculation, we fixed $g, H_{c}$, and $U$, and fitted $J_{0}$ according to Eq. (30).

Corresponding calculations for $\Gamma_{H}(T)$ using Eqs. (28) and (44) are shown in Fig. 2 and for $S(T)$ in Fig. 3, while in Fig. 4, we display a series of isoentropic lines for $\mathrm{Ba}_{3} \mathrm{Cr}_{2} \mathrm{O}_{8}$ and $\mathrm{Sr}_{3} \mathrm{Cr}_{2} \mathrm{O}_{8}$. The phase transition is clearly visible in all these figures. The Grüneisen parameter $\Gamma_{H}(T)$ shows a discontinuity according to Eq. (70) and changes its sign at $T_{c}(H)$, while the entropy $S(T)$ exhibits a change in its slope, thereby reflecting a discontinuity in the heat capacity $C_{H}$ according to Eq. (67).

The isoentropic lines shown in Fig. 4 have a minimum at $H_{c}(T)$, which can be easily understood by recalling that
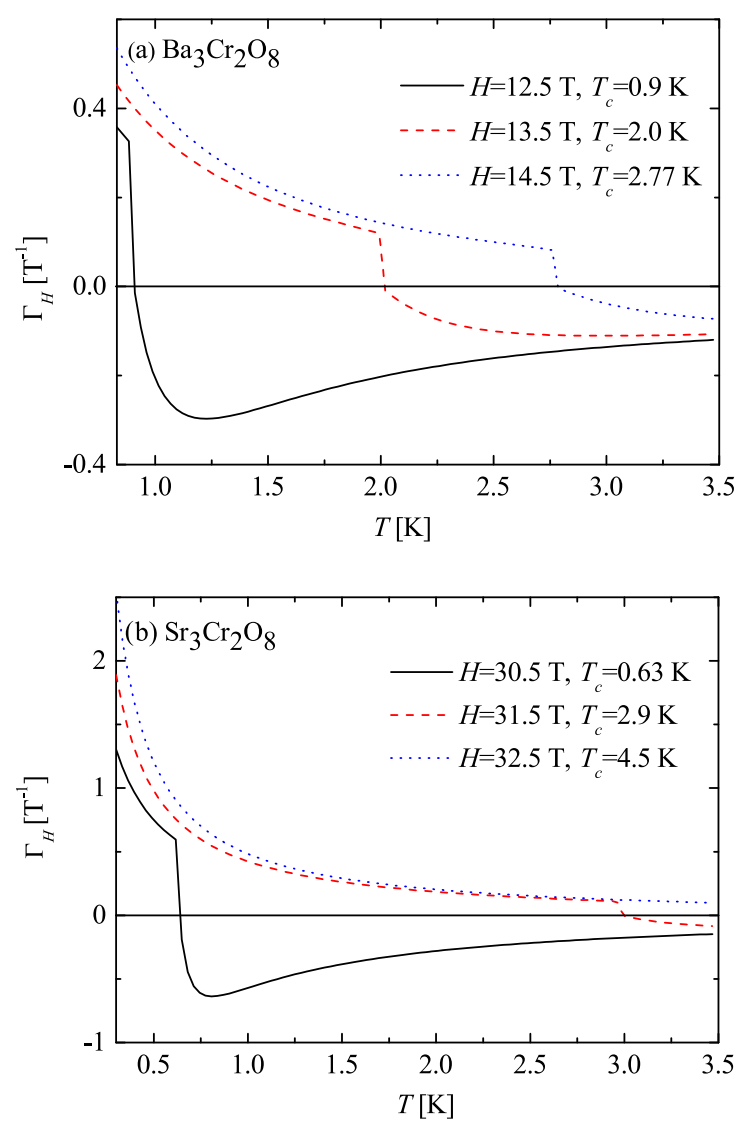

FIG. 2. The dependence of the Grüneisen parameter on temperature for (a) $\mathrm{Ba}_{3} \mathrm{Cr}_{2} \mathrm{O}_{8}$ and (b) $\mathrm{Sr}_{3} \mathrm{Cr}_{2} \mathrm{O}_{8}$ in different magnetic fields. At the respective $T_{c}, \Gamma_{H}(T)$ shows a sign-changing discontinuity. 

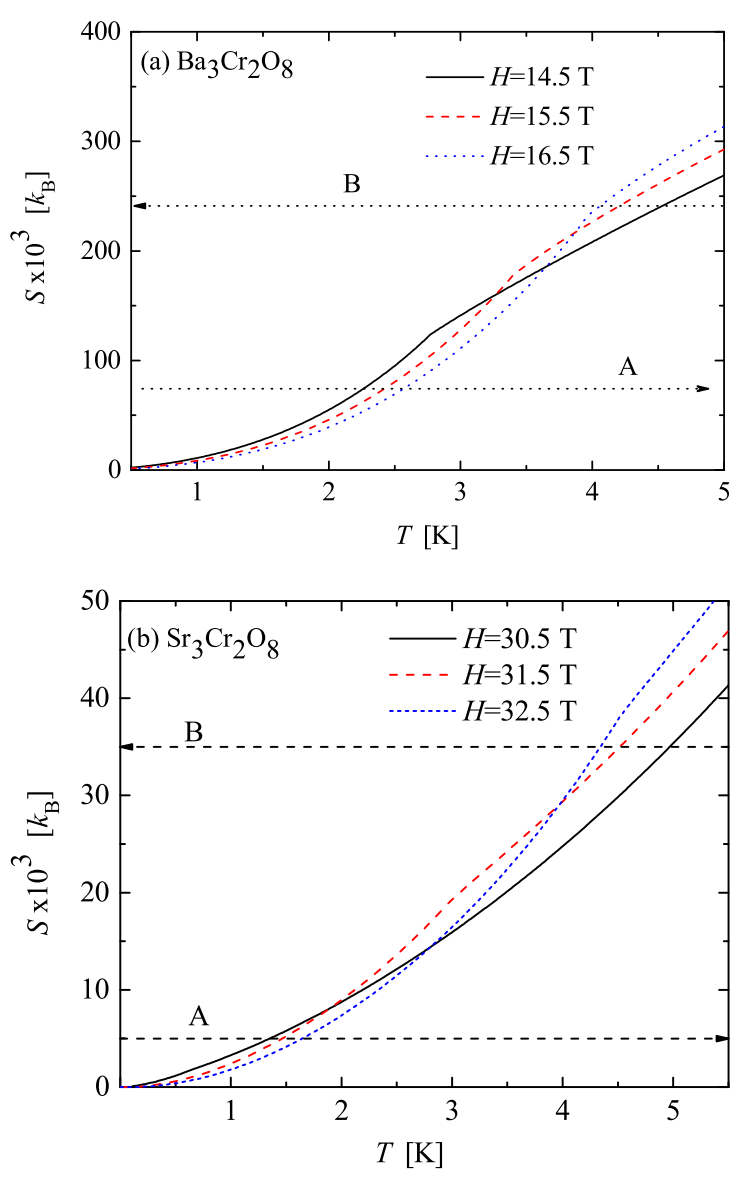

FIG. 3. The entropy $S$ vs temperature $T$ for $\mathrm{Ba}_{3} \mathrm{Cr}_{2} \mathrm{O}_{8}$ and $\mathrm{Sr}_{3} \mathrm{Cr}_{2} \mathrm{O}_{8}$ for different values of the magnetic field $H$. As expected, $S(T)$ changes the slope at $T_{c}$. The line marked A shows a path along which adiabatic increasing of the magnetic field causes the temperature to increase. The line marked $\mathrm{B}$ shows a path along which the same procedure causes the temperature to decrease. For convenience, we restored here and in Fig. 4 the Boltzmann constant $k_{\mathrm{B}}$.

$\Gamma_{H}=T^{-1}(\partial T / \partial H)_{S}$ vanishes at the phase transition. In a perfectly adiabatic experiment, the temperature would ideally follow these lines upon a change of the external magnetic field, reaching its lowest temperature at $H_{c}$. The diagram shown in Fig. 4 for $\mathrm{Sr}_{3} \mathrm{Cr}_{2} \mathrm{O}_{8}$ compares favorably with that measured by Aczel et al. [43]. We note that in most conventional magnetocaloric experiments, a sample is subject to a controlled heat link, so that the corresponding $T(H)$ curves become time dependent $[9,43,47,48]$ and change their shape in comparison with those displayed in Fig. 4.

\section{MEASURABILITY}

While the discontinuities in $C_{H}$ and $\Gamma_{H}$ [Eqs. (67) and (68)] and the sign change in $\Gamma_{H}$ at $T_{c}$ can, in principle, be directly measured in a dedicated experiment, an examination of the temperature dependence of these quantities in the lowtemperature limit may face the problem that the heat capacity $C_{H}$ of the magnetic subsystem exhibits the same temperature dependence as that of the crystal lattice, i.e., $C_{H} \sim T^{3}$, and the magnetic contribution has then to be extracted from the total signal. This is possible, e.g., by performing a series of
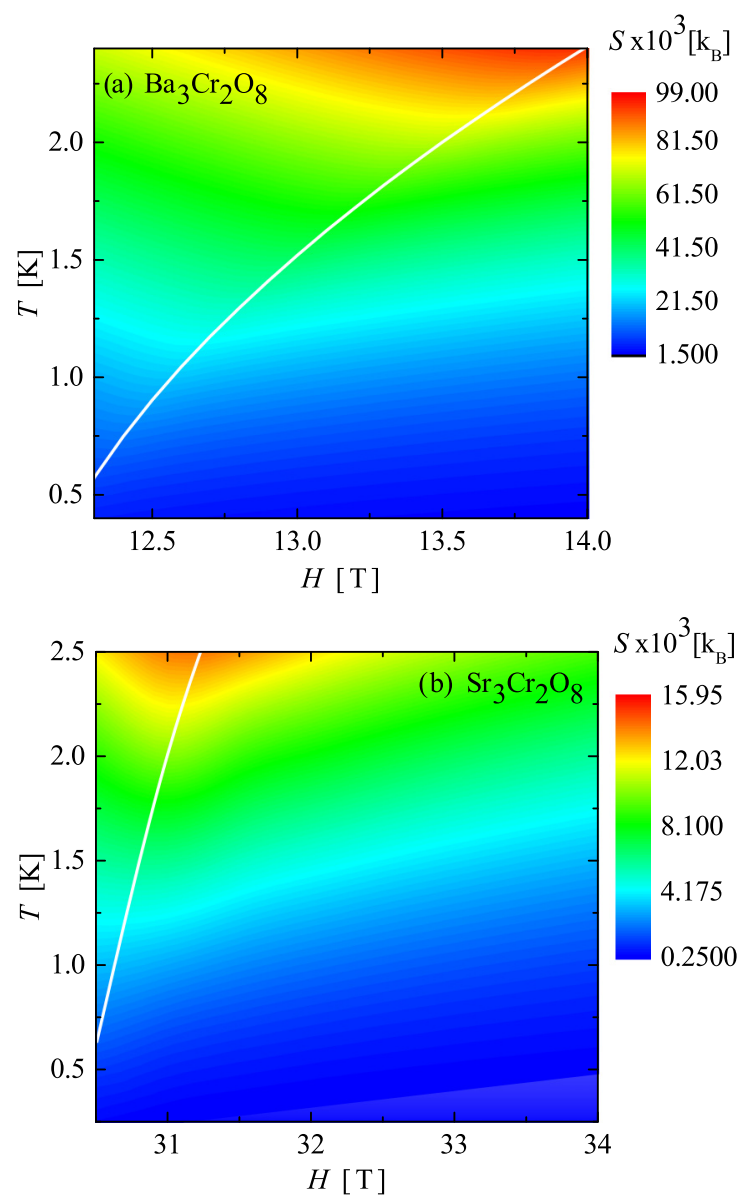

FIG. 4. Isoentropic lines for the magnetic system of (a) $\mathrm{Ba}_{3} \mathrm{Cr}_{2} \mathrm{O}_{8}$ and (b) $\mathrm{Sr}_{3} \mathrm{Cr}_{2} \mathrm{O}_{8}$ in the $(H, T)$ plane. Each color corresponds to a constant entropy value. The white lines show the phase boundaries separating the condensed (right side) from the uncondensed (left side) phases, respectively.

measurements in different magnetic fields (and in $H=0$ in the case of $C_{H}$ ) provided that the lattice heat capacity $C_{\text {lat }}$ does not entirely dominate $C_{H}$. For $N_{\text {at }}$ atoms in the unit cell, we have in the low-temperature limit the Debye result per unit cell:

$$
C_{\text {lat }} \approx N_{\mathrm{at}} \frac{12 \pi^{4} T^{3}}{5 T_{\mathrm{D}}^{3}},
$$

with the Debye temperature of the lattice $T_{\mathrm{D}}$. Since the heat capacity of the magnetic subsystem is proportional to the number of spin dimers $N_{\text {dim }}<N_{\text {at }}$, the ratio of the two contributions is

$$
\frac{C_{H}}{C_{\text {lat }}}=\frac{1}{18 \pi^{2}} \frac{N_{\text {dim }}}{N_{\text {at }}} \frac{T_{\mathrm{D}}^{3}}{c_{0}^{3}},
$$

where the speed of sound from Eq. (35) is expressed in Kelvin. Due to a very small factor $1 / 18 \pi^{2}$, this ratio seems to be unfavorable small. However, by performing an experiment close enough to the transition one can force $c \ll T_{\mathrm{D}}$, and the two contributions may become separable. The qualitative field dependence $\Gamma_{H} \sim\left(H-H_{c}\right)^{-1}$ from Eq. (42) and the magnetizations (55), (56), and (71) remain unaffected by these arguments and should be readily accessible in a corresponding 
experiment, while the absolute value of the measured $\Gamma_{H}$ has to be corrected for the contribution of $C_{\text {lat }}$ to Eq. (3). To give an example of the parameters involved, with $J_{0}=15$ $\mathrm{K}$ and $g \approx 2$ as for $\mathrm{Sr}_{3} \mathrm{Cr}_{2} \mathrm{O}_{8}$, we estimate $c_{0} \approx 4.5 \mathrm{~K}$ for $H-H_{c}=1 \mathrm{~T}$, so that with $N_{\mathrm{dim}} / N_{\mathrm{at}}=1 / 13$ (for a single $\mathrm{Cr}-\mathrm{Cr}$ dimer bond per 13 atoms in the unit cell) and $T_{\mathrm{D}} \approx 120$ $\mathrm{K}$ [49], $C_{H} / C_{\text {lat }} \approx 8$.

\section{CONCLUSIONS}

We have performed a mean field variational Gaussianapproximation analysis of gapped dimerized quantum magnets showing a Bose-Einstein condensation of magnetic quasiparticles such as triplons. We calculated the free energy $\Omega$ and the associated entropy $S$, the heat capacity $C_{H}$, the magnetization $M$, and the Grüneisen parameter $\Gamma_{H}$, and derived explicit expressions for these quantities in the limits $T \rightarrow T_{c}$ and $T \rightarrow 0$, respectively. Near the critical temperature, both the heat capacity and the Grüneisen parameter show a discontinuity with $\Gamma_{H}$ changing the sign. Such a behavior is expected for systems with a magnetically controlled quantum critical point [5]. In the low-temperature limit near the transition, we find that $C_{H} \sim T^{3}$, which is universal for Bose condensed interacting systems. At low temperatures, the Grüneisen parameter diverges as $\Gamma_{H} \sim T^{-2}$. Approaching the transition field as $H \rightarrow H_{c}$ we find $\Gamma_{H} \sim G_{r}\left(H-H_{c}\right)^{-1}$, common to a variety of magnetic systems [7]. The parameter $G_{r}$ reaches its universal value $G_{r} \rightarrow 1 / 2$ in the unitary limit when the $s$-wave scattering length greatly exceeds the effective lattice constant, $a_{s} \gg a$. Corresponding experiments to verify these conjectures and compare them with the scalings predicted by the full quantum theories of phase transitions should be feasible.

\section{ACKNOWLEDGMENTS}

This work is partially supported by the Swiss National Foundation SCOPES project IZ74Z0_160527 and by the Academy of Science of the Republic of Uzbekistan project $\Phi A-\Phi 2-004$. A. Gazizulina is supported by the Swiss National Science Foundation Grant No. 21-153659. E.Y.S. acknowledges support by the Spanish Ministry of Economy, Industry and Competitiveness (MINECO) and the European Regional Development Fund FEDER through Grant No. FIS2015-67161-P (MINECO/FEDER, UE), and the Basque Government through Grant No. IT986-16. Z.N. thanks the Academy of Science of the Republic of Uzbekistan for funding through Grant No. FPFI $\Phi .2-18$. We are obliged to P. Gegenwart for useful discussions.

\section{APPENDIX A: FREE ENERGY}

Here we derive the free energy given in (11) by using a variational perturbative theory. This perturbative scheme includes the following steps:

(1) We parametrize the quantum filed $\psi$ in terms of a timeindependent condensate $\rho_{0}$ and a quantum fluctuation field $\widetilde{\psi}$ as

$$
\psi=\sqrt{\rho_{0}}+\widetilde{\psi}
$$

which defines the number of uncondensed particles as

$$
\rho_{1}=\int d^{3} r\left\langle\widetilde{\psi}^{\dagger} \widetilde{\psi}\right\rangle
$$

where the expectation value of an operator $\left\langle\hat{O}\left(\widetilde{\psi}^{\dagger}, \widetilde{\psi}\right)\right\rangle$ is defined as

$$
\langle\hat{O}\rangle=\frac{1}{\mathcal{Z}} \int \mathcal{D} \widetilde{\psi}^{\dagger} \mathcal{D} \widetilde{\psi} \hat{O}\left(\widetilde{\psi}^{\dagger}, \widetilde{\psi}\right) e^{-\mathcal{A}\left[\widetilde{\psi}^{\dagger}, \widetilde{\psi}\right]} .
$$

Then the total number of particles is given by

$$
\rho=\rho_{1}+\rho_{0} .
$$

(2) We replace $U$ in (8) as $U \rightarrow \delta U$ and add to (8) the following term:

$$
S_{\Sigma}=(1-\delta) \int d \tau d^{3} r\left[\Sigma_{\mathrm{n}} \tilde{\psi}^{\dagger} \widetilde{\psi}+\frac{1}{2} \Sigma_{\mathrm{an}}\left(\widetilde{\psi}^{\dagger} \widetilde{\psi}^{\dagger}+\widetilde{\psi} \widetilde{\psi}\right)\right]
$$

where the variational parameters $\Sigma_{\mathrm{n}}$ and $\Sigma_{\text {an }}$ may be interpreted as the normal and the anomalous self energies, respectively.

(3) Now the perturbation scheme may be considered as an expansion in powers of $\delta$ by using the propagators

$$
G_{a b}\left(\tau, r ; \tau^{\prime}, r^{\prime}\right)=\frac{1}{\beta} \sum_{n, \mathbf{k}} e^{i \omega_{n}\left(\tau-\tau^{\prime}\right)+i \mathbf{k}\left(\mathbf{r}-\mathbf{r}^{\prime}\right)} G_{a b}\left(\omega_{n}, \mathbf{k}\right)
$$

$(a, b=1,2)$, where $\omega_{n}=2 \pi n T$ is the $n$th bosonic Matsubara frequency, $\sum_{n, \mathbf{k}}=\sum_{n=-\infty}^{\infty} \int d^{3} k /(2 \pi)^{3}$, and

$$
G_{a b}\left(\omega_{n}, \mathbf{k}\right)=\frac{1}{\omega_{n}^{2}+E_{\mathbf{k}}^{2}}\left(\begin{array}{lr}
\varepsilon_{\mathbf{k}}+X_{2} & \omega_{n} \\
-\omega_{n} & \varepsilon_{\mathbf{k}}+X_{1}
\end{array}\right) .
$$

In (A7) $E_{\mathbf{k}}$ corresponds to the dispersion of quasiparticles

$$
E_{\mathbf{k}}=\sqrt{\varepsilon_{\mathbf{k}}+X_{1}} \sqrt{\varepsilon_{\mathbf{k}}+X_{2}},
$$

where $X_{1}$ and $X_{2}$, given by

$$
\begin{aligned}
& X_{1}=\Sigma_{\mathrm{n}}+\Sigma_{\mathrm{an}}-\mu, \\
& X_{2}=\Sigma_{\mathrm{n}}-\Sigma_{\mathrm{an}}-\mu
\end{aligned}
$$

may be considered as variational parameters instead of $\Sigma_{\mathrm{n}}, \Sigma_{\mathrm{an}}$. The parameter $\delta$ should be set $\delta=1$ at the end of the calculations. This perturbation scheme is known as the $\delta$-expansion method [50].

(4) After subtraction of discontinuous and one-particle reducible diagrams, we obtain the free energy $\Omega$ as a function of $\rho_{0}, X_{1}$, and $X_{2}$.

The variational parameters $X_{1}$ and $X_{2}$ may be fixed by the requirements

$$
\frac{\partial \Omega\left(X_{1}, X_{2}, \rho_{0}\right)}{\partial X_{1}}=\frac{\partial \Omega\left(X_{1}, X_{2}, \rho_{0}\right)}{\partial X_{2}}=0 .
$$

Provided Eqs. (A10) are satisfied, the condensed density $\rho_{0}$ it is determined by the stationary condition

$$
\frac{\partial \Omega}{\partial \rho_{0}}=0 .
$$

Note that (A11) is equivalent to the condition $\langle\widetilde{\psi}\rangle=0$, which is obtained by the requirement $H^{(1)}\left(\widetilde{\psi}, \widetilde{\psi}^{\dagger}\right)=0$ in the 
Hamiltonian formalism [51], where $H^{(1)}$ is the part of the Hamiltonian which is linear at $\widetilde{\psi}$. The accuracy of the $\delta$ expansion to calculate $\Omega$ is somewhat limited by the fact that the inclusion of loop integrals, which complicate the calculation process $[27,52]$, is not carried out here.

\section{APPENDIX B: NORMAL AND ANOMALOUS DENSITIES}

Here we present explicit expressions for $\mathcal{E}_{\mathbf{k}, T}^{\prime}=\partial \mathcal{E}_{\mathbf{k}} / \partial T$ and $\mathcal{E}_{\mathbf{k}, \mu}^{\prime}=\partial \mathcal{E}_{\mathbf{k}} / \partial \mu$, which are needed for the evaluation of the entropy and heat capacity in equations (20)-(22). Then we present the details of the low temperature expansion exploited in Sec. III. In the normal phase when $\mathcal{E}_{\mathbf{k}}=\omega_{\mathbf{k}}=\varepsilon_{\mathbf{k}}-\mu+$ $2 U \rho$, the density of particles is given by

$$
\rho=\sum_{\mathbf{k}} f_{B}\left(\omega_{\mathbf{k}}\right)
$$

where $f_{B}(x)=1 /\left(e^{\beta x}-1\right)$. Clearly,

$$
\frac{\partial \omega_{\mathbf{k}}}{\partial T}=2 U \frac{\partial \rho}{\partial T},
$$

which does not depend on momentum $k$. Differentiating both sides of the equation (B1) with respect to $T$, we find

$$
\begin{aligned}
\frac{\partial \rho}{\partial T} & =\frac{\beta S_{1}}{2 S_{2}-1}, \\
S_{1} & =-\beta \sum_{\mathbf{k}} \omega_{\mathbf{k}} f_{B}^{2}\left(\omega_{\mathbf{k}}\right) e^{\beta \omega_{\mathbf{k}}}, \\
S_{2} & =-U \beta \sum_{\mathbf{k}} f_{B}^{2}\left(\omega_{\mathbf{k}}\right) e^{\beta \omega_{\mathbf{k}}} .
\end{aligned}
$$

Taking the derivative with respect to $\mu$ gives

$$
\begin{gathered}
\frac{\partial \omega_{\mathbf{k}}}{\partial \mu}=2 U \frac{\partial \rho}{\partial \mu}-1, \\
\frac{\partial \rho}{\partial \mu}=\frac{S_{2}}{U\left(2 S_{2}-1\right)} .
\end{gathered}
$$

In the condensed phase, $T<T_{c}, \mathcal{E}_{\mathbf{k}}=E_{\mathbf{k}}=\sqrt{\varepsilon_{\mathbf{k}}\left(\varepsilon_{\mathbf{k}}+2 \Delta\right)}$, and hence we have

$$
\begin{aligned}
\frac{\partial E_{\mathbf{k}}}{\partial T} & =\frac{\varepsilon_{\mathbf{k}}}{E_{\mathbf{k}}} \Delta_{T}^{\prime}, \\
\frac{\partial E_{\mathbf{k}}}{\partial \mu} & =\frac{\varepsilon_{\mathbf{k}}}{E_{\mathbf{k}}} \Delta_{\mu}^{\prime} \\
\frac{\partial \rho}{\partial T} & =\frac{\Delta_{T}^{\prime}}{2 U} .
\end{aligned}
$$

To find, e.g., $\Delta_{T}^{\prime}$ we can differentiate both sides of the equation (36) with respect to $T$ and solve it with respect to $\Delta_{T}^{\prime}$. The results are

$$
\begin{aligned}
\Delta_{T}^{\prime} & =\frac{\partial \Delta}{\partial T}=\frac{U S_{4}}{2 T\left(2 S_{5}+1\right)}, \\
\Delta_{\mu}^{\prime} & =\frac{\partial \Delta}{\partial \mu}=\frac{1}{2 S_{5}+1}, \\
S_{4} & =\sum_{\mathbf{k}} W_{\mathbf{k}}^{\prime}\left(\varepsilon_{\mathbf{k}}+2 \Delta\right),
\end{aligned}
$$

$$
\begin{aligned}
S_{5} & =U \sum_{\mathbf{k}} \frac{4 W_{\mathbf{k}}+E_{\mathbf{k}} W_{\mathbf{k}}^{\prime}}{4 E_{\mathbf{k}}}, \\
W_{\mathbf{k}}^{\prime} & =\beta\left(1-4 W_{\mathbf{k}}^{2}\right), \\
W_{\mathbf{k}} & =\frac{1}{2}+f_{B}\left(E_{\mathbf{k}}\right) .
\end{aligned}
$$

Below we illustrate the low-temperature expansion explicitly. For this purpose we follow the strategy outlined in Sec. III and start with $\rho_{1}$. Equation (38) may be rewritten as

$$
\rho_{1}=\sum_{\mathbf{q}} \frac{\varepsilon_{q}+\Delta}{E_{q}\left(\exp \left(E_{q} \beta\right)-1\right)}+\rho_{1}(0),
$$

and its $T$-dependent part as

$$
\begin{aligned}
\rho_{1}(T)-\rho_{1}(0) & =\sum_{\mathbf{q}} \frac{\varepsilon_{q}+\Delta}{E_{q}\left(\exp \left(E_{q} \beta\right)-1\right)} \\
& =\frac{1}{4 m c_{0}} \int_{0}^{Q} d q \frac{q\left(q^{2} \pi^{2}+2 m^{2} c_{0}^{2}\right)}{\exp \left(\pi c_{0} q \beta\right)-1} .
\end{aligned}
$$

At small $T$, we can perform a low-temperature expansion and obtain

$$
\rho_{1}(T)-\rho_{1}(0)=\frac{m T^{2}}{12 c_{0}}+\frac{\pi^{2} T^{4}}{60 m c_{0}^{5}}+O(z),
$$

where $z=\exp \left(-\pi Q c_{0} / T\right)$. Therefore,

$$
\rho_{1}(T)=\rho_{1}(0)+\frac{\widetilde{T}^{2}}{12 \gamma}+O\left(\widetilde{T}^{4}\right),
$$

where $\gamma=m c_{0}$. The low temperature expansion for the key quantity $\Delta_{T}^{\prime}$, given in (51), can be evaluated in the similar way.

Now integrating the relation $\rho_{T}^{\prime}=\Delta_{T}^{\prime} / 2 U$ over $T$ and using (51) one may find the low temperature expansion for the total triplon density as

$$
\rho(T)=\rho(0)-\frac{\alpha_{1}}{4 U \gamma m} \widetilde{T}^{2}+O\left(\widetilde{T}^{4}\right) .
$$

The previous two equations yield for the condensed fraction

$$
\rho_{0}(T)=\rho(T)-\rho_{1}(T)=\rho_{0}(0)-\frac{3 \alpha_{1}+U m}{12 U m \gamma} \widetilde{T}^{2}+O\left(\widetilde{T}^{4}\right) .
$$

Finally, excluding $\Delta$ from equations (43) gives the low temperature expansion for the anomalous density

$$
\sigma(T)=\sigma(0)+\frac{U m-3 \alpha_{1}}{12 U m \gamma} \widetilde{T}^{2}+O\left(\widetilde{T}^{4}\right) .
$$

\section{APPENDIX C: $G_{r}$ AND $G_{t}$ PARAMETERS}

Here we will show that if $r=\left(H-H_{c}\right) / H_{c}=\mu / \Delta_{\text {st }}$ is small (where $\Delta_{\mathrm{st}}=g \mu_{B} H_{c}$ is the spin gap), the Grüneisen parameter diverges as $\Gamma_{H} \sim 1 / r$ at low temperatures. We shall derive also explicit expressions for $G_{r}$ and $G_{t}$ given in Eqs. (72) and (73).

For this purpose we rewrite the equation (54) as:

$$
\Gamma_{H}=\gamma_{0}+\frac{\gamma_{2}}{T^{2}}
$$


with

$$
\begin{aligned}
& \gamma_{0}=\frac{g \mu_{B}\left(6 U Q \gamma \pi+6 \pi^{2} c_{0}+5 U \gamma^{2}-10 U \gamma^{4}\right)}{12 \gamma^{3}\left(U Q \gamma+\pi c_{0}\right)^{2}}, \\
& \gamma_{2}=\frac{5 g \mu_{B} \gamma^{3}}{2 \pi\left(U Q \gamma+\pi c_{0}\right)}
\end{aligned}
$$

where $c_{0}^{2}=\Delta_{0} / m$, as in Eq. (47). First, we show that $c_{0}=$ $\tilde{c} \sqrt{r}+O\left(r^{3 / 2}\right)$, where $r$-independent $\widetilde{c}$ will be obtained below.

The value of $\Delta_{0}$ determined by Eq. (36), where $\sigma$ and $\rho_{1}$ are taken from (37) and (38) with $W_{\mathbf{k}}=1 / 2$, can be simplified as

$$
\Delta_{0}=\mu+U \sum_{\mathbf{k}}\left(1-\frac{E_{\mathbf{k}}}{\varepsilon_{\mathbf{k}}}\right)
$$

with $E_{\mathbf{k}}=\sqrt{\varepsilon_{\mathbf{k}}} \sqrt{\varepsilon_{\mathbf{k}}+2 \Delta_{0}}$. In the spherical Debye-like approximation, the momentum integration in (C3) can be taken explicitly, even without linear approximation for $E_{\mathbf{k}}$, resulting in

$$
\Delta_{0}=\mu+\frac{U}{6 \pi^{2}}\left[8\left(\Delta_{0} m\right)^{3 / 2}+\pi^{3} Q^{3}-\left(\pi^{2} Q^{2}+4 m \Delta_{0}\right)^{3 / 2}\right] .
$$

Since it is expected that at $r \rightarrow 0$ the quantity $m \Delta_{0} \ll 1$ we can expand RHS of (C4) in powers of $m \Delta_{0}$ to obtain

$$
\Delta_{0}=\mu-\frac{U m \Delta_{0} Q}{\pi}+O\left(\left(m \Delta_{0}\right)^{3 / 2}\right)
$$

or in terms of $r$ as

$$
\Delta_{0} \approx r \Delta_{\mathrm{st}}-\frac{U m \Delta_{0} Q}{\pi} .
$$

Thus for small $r$ the $\Delta_{0}$ is given by the solution of this linear equation, $\Delta_{0} \approx r \Delta_{\mathrm{st}} \pi /(\pi+U m Q)$, and hence

$$
\tilde{c}=\sqrt{\frac{\pi \Delta_{\mathrm{st}}}{m(\pi+U m Q)}}=\sqrt{\frac{\pi g \mu_{B} H_{c}}{m(\pi+U m Q)}} .
$$

Inserting this expression into $(\mathrm{C} 2)$ gives

$$
\begin{gathered}
\gamma_{0}=\frac{2 g \mu_{B}}{U Q^{2} \widetilde{c}^{2} m^{2} r}+O\left(r^{-1 / 2}\right), \\
\gamma_{2}=\frac{10 g \mu_{B} \widetilde{c}^{2} r}{\pi^{2} Q^{2} U}+O\left(r^{3 / 2}\right) .
\end{gathered}
$$

Finally using here (C7) one obtains

$$
\begin{gathered}
G_{r} \equiv \gamma_{0}\left(H-H_{c}\right) \approx \frac{2}{\pi Q}+\frac{2}{U m Q^{2}}, \\
G_{t} \equiv \frac{\gamma_{2}}{H-H_{c}} \approx \frac{10 \mu_{B}^{2} g^{2}}{\pi m U Q^{2}(\pi+U m Q)},
\end{gathered}
$$

as presented in Sec. V.
[1] P. Debye, Ann. Phys. 386, 1154 (1926).

[2] W. F. Giauque, J. Am. Chem. Soc. 49, 1864 (1927).

[3] B. Wolf, A. Honecker, W. Hofstetter, U. Tutsch, and M. Lang, Int. J. Mod. Phys. B 28, 1430017 (2014).

[4] L. Zhu, M. Garst, A. Rosch, and Q. Si, Phys. Rev. Lett. 91, 066404 (2003).

[5] M. Garst and A. Rosch, Phys. Rev. B 72, 205129 (2005).

[6] Y. Tokiwa and P. Gegenwart, Rev. Sci. Instrum. 82, 013905 (2011).

[7] P. Gegenwart, Philos. Mag. 97, 3415 (2017).

[8] P. Gegenwart, Rep. Prog. Phys. 79, 114502 (2016).

[9] V. Zapf, M. Jaime, and C. D. Batista, Rev. Mod. Phys. 86, 563 (2014).

[10] T. Giamarchi, C. Ruegg, and O. Tchernyshyov, Nat. Phys. 4, 198 (2008).

[11] F. Weickert, R. Küchler, A. Steppke, L. Pedrero, M. Nicklas, M. Brando, F. Steglich, M. Jaime, V. S. Zapf, A. Paduan-Filho, K. A. Al-Hassanieh, C. D. Batista, and P. Sengupta, Phys. Rev. B 85, 184408 (2012).

[12] S. A. Zvyagin, J. Wosnitza, C. D. Batista, M. Tsukamoto, N. Kawashima, J. Krzystek, V. S. Zapf, M. Jaime, N. F. Oliveira Jr., and A. Paduan-Filho, Phys. Rev. Lett. 98, 047205 (2007).

[13] T. Nikuni, M. Oshikawa, A. Oosawa, and H. Tanaka, Phys. Rev. Lett. 84, 5868 (2000).
[14] T. Radu, H. Wilhelm, V. Yushankhai, D. Kovrizhin, R. Coldea, Z. Tylczynski, T. Lühmann, and F. Steglich, Phys. Rev. Lett. 95, 127202 (2005).

[15] A. Rakhimov, E. Ya. Sherman, and C. K. Kim, Phys. Rev. B 81, 020407(R) (2010).

[16] A. Rakhimov, S. Mardonov, and E. Ya. Sherman, Ann. Phys. 326, 2499 (2011).

[17] A. Rakhimov, S. Mardonov, E. Ya. Sherman, and A. Schilling, New J. Phys. 14, 113010 (2012).

[18] A. Khudoyberdiev, A. Rakhimov, and A. Schilling, New J. Phys. 19, 113002 (2017).

[19] J. Andersen, Rev. Mod. Phys. 76, 599 (2004).

[20] T. Matsubara and H. Matsuda, Prog. Theor. Phys. 16, 569 (1956).

[21] M. Matsumoto, B. Normand, T. M. Rice, and M. Sigrist, Phys. Rev. B 69, 054423 (2004).

[22] G. Misguich and M. J. Oshikawa, J. Phys. Soc. Jpn. 73, 3429 (2004).

[23] E. Ya. Sherman, P. Lemmens, B. Busse, A. Oosawa, and H. Tanaka, Phys. Rev. Lett. 91, 057201 (2003).

[24] The repulsion $U$ can be considered as a phenomenological parameter, needed to obtain an agreement between theory and experiment. An example of a model calculation of its value in terms of a magnon-magnon scattering amplitude can be found in D. Yamamoto, G. Marmorini, and I. Danshita, Phys. Rev. Lett. 112, 127203 (2014). 
[25] H. Kleinert, Z. Narzikulov, and A. Rakhimov, Phys. Rev. A 85, 063602 (2012).

[26] M. Le Bellac, Thermal Field Theory (Cambridge University Press, Cambridge, 1996).

[27] H. Kleinert and V. Schulte-Frohlinde, Critical Phenomena in $\phi^{4}$-Theory (World Scientific, Singapore, 2001).

[28] H. Kleinert, Z. Narzikulov, and A. Rakhimov, J. Stat. Mech. (2014) P01003.

[29] A. Rakhimov, C. K. Kim, S.-H. Kim, and J. H. Yee, Phys. Rev. A 77, 033626 (2008).

[30] V. I. Yukalov and H. Kleinert, Phys. Rev. A 73, 063612 (2006).

[31] F. Yamada, T. Ono, H. Tanaka, G. Misguich, M. Oshikawa, and T. Sakakibara, J. Phys. Soc. Jpn. 77, 013701 (2008).

[32] N. M. Hugenholtz and D. Pines, Phys. Rev. 116, 489 (1959).

[33] For the triplon BEC of our interest, the bare dispersion $\varepsilon_{\mathbf{k}}$ is counted from the bottom of the Zeeman-split lower triplet band.

[34] T. Haugset, H. Haugerud, and F. Ravndal, Ann. Phys. 266, 27 (1998).

[35] R. DellAmore, A. Schilling, and K. Kramer, Phys. Rev. B 79, 014438 (2009).

[36] V. I. Yukalov, Laser Physics 19, 1 (2009) (see Chap. 7).

[37] J. E. Robinson, Phys. Rev. 83, 678 (1951).

[38] L. Pitaevskii and S. Stringari, Bose-Einstein Condensation and Superfluidity (Oxford University Press, Oxford, 2016).

[39] A. Fetter and J. Walecka, Quantum Theory of Many Particle System (Dover Publications, Inc., Mineola, NY, 2003).

[40] A. Rakhimov and I. N. Askerzade, Int. J. Mod. Phys. B 29, 1550123 (2015); Phys. Rev. E 90, 032124 (2014).
[41] K. Huang, Statistical Physics (John Wiley \& Sons, Hoboken, NJ, 1997).

[42] Z. Yao, K. P. C. da Costa, M. Kiselev, and N. Prokof'ev, Phys. Rev. Lett. 112, 225301 (2014).

[43] A. A. Aczel, Y. Kohama, C. Marcenat, F. Weickert, M. Jaime, O. E. Ayala-Valenzuela, R. D. McDonald, S. D. Selesnic, H. A. Dabkowska, and G. M. Luke, Phys. Rev. Lett. 103, 207203 (2009).

[44] M. Kofu, H. Ueda, H. Nojiri, Y. Oshima, T. Zenmoto, K. C. Rule, S. Gerischer, B. Lake, C. D. Batista, Y. Ueda, and S.-H. Lee, Phys. Rev. Lett. 102, 177204 (2009).

[45] Z. Wang, D. L. Quintero-Castro, S. Zherlitsyn, S. Yasin, Y. Skourski, A. T. M. N. Islam, B. Lake, J. Deisenhofer, and A. Loidl, Phys. Rev. Lett. 116, 147201 (2016).

[46] H. Tanaka, F. Yamada, T. Ono, T. Sakakibara, Y. Uwatoko, A. Oosawa, K. Kakurai, and K. Goto, J. Magn. Magn. Mater. 310, 1343 (2007).

[47] Y. Kohama, Ch. Marcenat, T. Klein, and M. Jaime, Rev. Sci. Instrum. 81, 104902 (2010).

[48] A. Schilling and M. Reibelt, Rev. Sci. Instrum. 78, 033904 (2007).

[49] Z. Wang, Phys. Rev. B 85, 224304 (2012).

[50] F. F. deSouzaCruz, M. B. Pinto, and R. O. Ramos, Phys. Rev. B 64, 014515 (2001).

[51] H. T. C. Stoof, K. B. Gubbels, and D. B. M. Dickerscheid, Ultracold Quantum Fields (Springer, New York, 2009).

[52] I. Stancu and P. M. Stevenson, Phys. Rev. D 42, 2710 (1990). 